\title{
EFFECT OF AQUEOUS EXTRACT OF SORGHUM CROP RESIDUES ON WEED MANAGEMENT AND CROP PERFORMANCE OF WHEAT
}

\author{
F. Ahmed, M.R. Uddin*, M.D. Hossain, U.K. Sarker, D. Sarkar and D.N. Chadny \\ Department of Agronomy, Bangladesh Agricultural University, Mymensingh-2202, Bangladesh \\ *Corresponding Author, Email: romijagron@bau.edu.bd
}

(Received: 12 February 2019, Accepted: 10 April 2019)

Keywords: Wheat varieties, number of weeds, inhibition (\%), yield and harvest index (\%)

\begin{abstract}
An experiment was conducted at the Agronomy Field Laboratory, Bangladesh Agricultural University, Mymensingh to evaluate the effect of aqueous extract of sorghum crop residues on weed management and crop performance of wheat. The experiment consisted of three varieties of wheat viz., BARI Gom-19, BARI Gom-21, BARI Gom-24 and five different levels of treatments such as no use of aqueous extract, aqueous extract of sorghum crop residues: $1: 20$ ratio $(\mathrm{w} / \mathrm{v}), 1: 30$ ratio $(\mathrm{w} / \mathrm{v})$, $1: 40$ ratio $(\mathrm{w} / \mathrm{v})$ and hand weeding. The experiment was laid out in a randomized complete block design with three replications. Seven weed species belonging to five families infested the experimental plots. Weed population, weed dry weight and percent inhibition of weed were significantly influenced by aqueous extract of sorghum crop residues and varieties. The maximum weed growth was noticed with the variety BARI Gom-19 and the minimum was found in the variety BARI Gom-21. The grain yield as well as the other yield contributing characters produced by BARI Gom-21 was the highest among the studied varieties. The highest percent inhibition for all of the weeds was found in hand weeding treatment. The second highest percent weed inhibition was occurred in aqueous extract of sorghum crop residues@ @ $1: 20$ ratio $(\mathrm{w} / \mathrm{v})$ treatment which was $51.81,51.10,52.90,55.58,75.27,73.83$ and 53.85 percent for bathua (Chenopodium album), mutha (Cyperus rotundus), durba (Cynodon dactylon), biskatali (Polygonum hydropiper), angta (Paspalum scrobiculatum), tit begun (Solanum torvum) and shama (Echinochloa crusgalli) respectively. The highest loss of grain yield was obtained where no aqueous extract of sorghum crop residues were used. The highest yield and yield attributes were observed where hand weeding is done followed by the application of aqueous extract of sorghum crop residues @ 1:20 ratio $(\mathrm{w} / \mathrm{v})$. Wheat var. BARI Gom-21 with all treatments produced the highest grain and straw yield among the treatment combination. The results of this study indicate that different amount of aqueous extract of sorghum crop residues showed potential activity to suppress weed growth and it has significant effect on the yield of wheat. Therefore, aqueous extract of sorghum crop residues might be used as an alternative way for weed management in effective and sustainable crop production.
\end{abstract}

\section{Introduction}

Wheat (Triticum aestivum L.) is one of the world's most commonly consumed cereal grains. In Bangladesh, wheat is the second most important cereal crop next to rice. Wheat yield in Bangladesh has increased with the introduction of high yielding varieties and by the use of weed controlling measurements. In 
2015, average yield of wheat was estimated as $3.09 \mathrm{t} \mathrm{ha}^{-1}$ which was $1.75 \%$ higher than that of 2014 (BBS, 2015)

But on an average grain yield is lower than many countries of the world. There are several reasons for yield losses in wheat but weed infestation is greater than the combined losses of insect pests and diseases and all other attributes. The traditional method of weed control is hand weeding which is very much laborious and time consuming. Herbicide is effective in controlling weeds alone or in combination with hand weeding but it is harmful for the nature (Ahmed et. al., 2005). Beside this another observation was that "herbicides in combination with hand weeding would help to obtain higher crop yield but it efforts high cost of production" (Prasad and Rafy, 1995; Sathyamoorthy et. al., 2004).

With rising human health and ecological concerns about the adverse effects of indiscriminate use of farm chemicals research on alternative weed management methods is underway worldwide. Exploitation of allelopathic potential of different crop/plant species for weed management under field conditions is one such approach. Crop allelopathy controls weeds by the release of allele-chemicals from the living plants and/or through decomposition of phytotoxic plant residues (Belz, 2004; Khan et. al., 2005). Sorghum [Sorghum bicolor (L.) Monech] is one of the strongest allelopathic crops which have been extensively used as a cover crop or through incorporation of its residue in soil to control weed. Sorghum is a successful competitor against weeds in fields as empirically known. Sorghum seems to have some inhibiting effects on the growth of weeds. These suggest the allelopathic potential of sorghum and it will be very useful for biological control of weeds.

Information regarding aqueous extract of sorghum crop residues for weed management is limited in our country. So, the study deserves to keep the significance in the current research interest in home and abroad about aqueous extract of sorghum crop residues having residual effects on weed control and yield performance to investigate the weed suppressing ability and to determine the optimum dose of aqueous extract of sorghum crop residues for the establishment of an easy, economic and sustainable method for efficient weed management and better yield of wheat.

\section{Materials and Methods}

The experiment was carried out at the Agronomy Field Laboratory of Bangladesh Agricultural University, Mymensingh from November 2016 to March 2017, located at $24^{\circ} 75^{\prime} \mathrm{N}$ latitude and $90^{\circ} 50^{\prime} \mathrm{E}$ longitude at an elevation of $18 \mathrm{~m}$ above the mean sea level characterized by non-calcareous dark grey floodplain soil belonging to the Old Brahmaputra Floodplain, (AEZ-9). The soil of the experimental field was more or less neutral in reaction with $\mathrm{pH}$ value 6.8, low in organic matter and fertility level. The land type was medium high with silty loam in texture. The experiment consists of two factors including crop residues (5) i) No use of extract (control), ii) Aqueous extract of sorghum crop residues @ $1: 20$ ratio $(\mathrm{w} / \mathrm{v})$, iii) Aqueous extract of sorghum crop residues @ 1:30 ratio $(w / v)$, iv) Aqueous extract of sorghum crop residues @ 1:40 ratio (w/v) and v) Hand weeding and wheat variety (3): i) BARI Gom-19 (Sourav) ii) BARI Gom-21 (Shatabdi) iii) BARI Gom-24 (Prodip). The seeds were sown on 21 November, 2016 as per treatment specifications. The experiment was laid out in a randomized complete block design with three replications. Thus total numbers of plots were 45. Each plot size was $(2 \mathrm{~m} \times 2.5 \mathrm{~m})$. The distance maintained between the individual plots was $0.5 \mathrm{~m}$ and the distance between the replication was $1.0 \mathrm{~m}$. 
After collection, the crop residues were dried under shade in the covered threshing floor of Agronomy Field Laboratory of BAU. The studied crop residues were cut as small as possible by using sickle. The small pieces of sorghum crop residues were dipped into water for 24 hours and then collected the aqueous extract from residues. The prepared sorghum aqueous extract was applied two times (20 days and 40 days) after seed sowing by a hand sprayer and harvesting was done in $8^{\text {th }}$ march, 2017.

Data were collected on the basis of different parameters of wheat and weeds. Among them percent inhibition shows the suppressing ability of aqueous extract of sorghum residues on weed.

Inhibition $(\%)=$ Dry weight of weed at control - Dry weight of weed from treatment Dry weight of weed at control

Data were also collected from wheat on yield basis such as grain yield, straw yield, harvest index etc which showed the yield performance of wheat. The recorded data were compiled and tabulated for statistical analysis. Analysis of variance was done with the help of computer package, MSTAT-C program. The mean differences among the treatments were adjudged by Duncan's Multiple Range Test.

\section{Results and Discussion}

\section{Infested weed species in the experimental field}

Seven weed species belonging to five families infested the experimental field. Local name, scientific name, family, morphological type and life cycle of the weed in the experimental plot have been presented in Table 1.

Table 2. Effect of variety on number and percent inhibition on different weeds

\begin{tabular}{|c|c|c|c|c|c|c|c|c|c|c|c|c|c|c|}
\hline \multicolumn{8}{|c|}{ Number of weed per quadrate $(25 \times 25) \mathrm{cm}^{2}$} & \multicolumn{7}{|c|}{$\%$ Inhibition } \\
\hline $\begin{array}{l}\text { Weed } \\
\text { name }\end{array}$ & Bathua & Mutha & Biskatali & Durba & $\begin{array}{c}\text { Tit } \\
\text { begun }\end{array}$ & Shama & Angta & Bathua & Mutha & Biskatali & Durba & $\begin{array}{c}\text { Tit } \\
\text { begun }\end{array}$ & Shama & Angta \\
\hline variety & & & & & & & & & & & & & & \\
\hline $\mathrm{v}_{1}$ & 6.11 & 5.86 & $7.33 b$ & 6.13 & $0.80 \mathrm{~b}$ & $6.80 a$ & $1.00 \mathrm{a}$ & $36.78 c$ & 40.06a & $38.45 b$ & 39.40 & $47.51 b$ & 37.38 & $\begin{array}{c}53.9 \\
1 \mathrm{~b}\end{array}$ \\
\hline $\mathrm{v}_{2}$ & 5.86 & 5.53 & $7.53 a$ & 6.07 & $1.00 \mathrm{a}$ & $6.26 b$ & $0.80 \mathrm{~b}$ & $38.97 a$ & $38.40 \mathrm{~b}$ & $39.62 a$ & 40.56 & $54.15 a$ & 39.29 & $\begin{array}{c}58.1 \\
9 a\end{array}$ \\
\hline $\mathrm{V}_{3}$ & 6.13 & 5.80 & $7.53 a$ & 6.27 & $1.00 \mathrm{a}$ & $6.60 \mathrm{ab}$ & $1.00 \mathrm{a}$ & $37.80 \mathrm{~b}$ & $37.42 b$ & $38.49 b$ & 40.60 & $52.53 a$ & 38.09 & $\begin{array}{c}54.2 \\
5 b\end{array}$ \\
\hline $\mathrm{S} \overline{\mathrm{x}}$ & 0.10 & 0.12 & 0.05 & 0.08 & 0.02 & 0.13 & 0.03 & 0.27 & 0.36 & 0.33 & 0.38 & 1.31 & 0.89 & 0.72 \\
\hline $\begin{array}{l}\text { CV } \\
(\%)\end{array}$ & 6.72 & 6.88 & 2.67 & 5.16 & 5.70 & 7.87 & 9.32 & 2.81 & 3.57 & 3.29 & 3.66 & 9.80 & 8.94 & 5.01 \\
\hline
\end{tabular}

$\mathrm{V}_{1}=$ BARI Gom-19 (Sourav), $\mathrm{V}_{2}=$ BARI Gom-21 (Shatabdi), $\mathrm{V}_{3}=$ BARI Gom-24 (Prodip)

\section{Effect of variety on number and percent inhibition on different weeds}

Variety shows the significant effect on number of weed population for biskatali, tit begun, shama and angta and others are not significantly affected. The lowest number of weeds was found in different varieties for different weeds (Table 2). On the other hand only shama and durba are not significant and rest of them are significantly affected in terms of percent inhibition. Bathua (38.97), tit begun (54.15), biskatali (39.62) and angta (58.19) shows highest percent inhibition for $\mathrm{V}_{2}$ and mutha (40.06) is found in $\mathrm{V}_{1}$ variety. (Table 2)

Table 1. Infested weed species found growing in the experimental plots in Wheat 


\begin{tabular}{|c|c|c|c|c|c|}
\hline $\begin{array}{l}\text { Sl. } \\
\text { No. }\end{array}$ & Local name & Scientific name & Family & $\begin{array}{l}\text { Morphological } \\
\text { type }\end{array}$ & Life cycle \\
\hline 1 & Mutha & $\begin{array}{l}\text { Cyperus } \\
\text { rotundus }\end{array}$ & Cyperaceae & Sedge & Perennial \\
\hline 2 & Durba & $\begin{array}{l}\text { Cynodon } \\
\text { dactylon }\end{array}$ & Poaceae & Grass & Perennial \\
\hline 3 & Bathua & $\begin{array}{l}\text { Chenopodium } \\
\text { album }\end{array}$ & Chenopodiaceae & Broad leaved & Annual \\
\hline 4 & Shama & $\begin{array}{l}\text { Echinochloa } \\
\text { crusgalli }\end{array}$ & Gramineae & Grass & Annual \\
\hline 5 & Angta & $\begin{array}{l}\text { Paspalum } \\
\text { scrobiculatum }\end{array}$ & Gramineae & Grass & Annual \\
\hline $\begin{array}{l}6 \\
7\end{array}$ & $\begin{array}{l}\text { Tit begun } \\
\text { Biskatali }\end{array}$ & $\begin{array}{l}\text { Solanum torvum } \\
\text { Polygonum } \\
\text { hydropiper }\end{array}$ & $\begin{array}{l}\text { Solanaceae } \\
\text { Polygonaceae }\end{array}$ & $\begin{array}{l}\text { Broad leaved } \\
\text { Broad leaved }\end{array}$ & $\begin{array}{l}\text { Perennial } \\
\text { Annual }\end{array}$ \\
\hline
\end{tabular}

Effect of aqueous extract of sorghum on number and percent inhibition on different weeds

Numbers of weed populations are significantly affected by the treatments for all weed species. Lowest weed population was found in $\mathrm{C}_{4}$ treatments (Hand weeding) followed by $\mathrm{C}_{1}$ treatment (Table 3). Highest percent inhibition was also found in $\mathrm{C}_{4}$ treatment which is followed by $\mathrm{C}_{1}$ treatment where the concentration of aqueous extract of sorghum was high (1:20). Numerically 51.81, 51.10, $55.58,52.90,73.83,53.85$ and 75.26 percent inhibition were found in bathua, mutha, biskatali, durba, tit begun, shama and angta respectively for $\mathrm{C}_{1}$ treatment. (Table 3)

Table 3. Effect of aqueous extract of sorghum on number and percent inhibition on different weeds

\begin{tabular}{|c|c|c|c|c|c|c|c|c|c|c|c|c|c|c|}
\hline \multicolumn{8}{|c|}{ Number of weed per quadrate $(25 \times 25) \mathrm{cm}^{2}$} & \multicolumn{7}{|c|}{ \% Inhibition } \\
\hline $\begin{array}{l}\text { Weed } \\
\text { name }\end{array}$ & Bathua & Mutha & Biskatali & Durba & $\begin{array}{c}\text { Tit } \\
\text { begun }\end{array}$ & Shame & Angta & Bathua & Mutha & Biskatali & Durba & $\begin{array}{c}\text { Tit } \\
\text { begun }\end{array}$ & Shama & Angta \\
\hline \multicolumn{15}{|c|}{ Treatments } \\
\hline $\mathrm{C}_{\mathrm{O}}$ & $8.78 \mathrm{a}$ & $8.33 a$ & $11.00 \mathrm{a}$ & $8.77 a$ & $1.99 \mathrm{a}$ & $9.55 a$ & $2.00 \mathrm{a}$ & $0.00 e$ & $0.00 e$ & $0.00 e$ & $0.00 e$ & $0.00 e$ & $0.00 e$ & $0.00 e$ \\
\hline $\mathrm{C}_{1}$ & $5.11 \mathrm{~d}$ & $4.66 \mathrm{~d}$ & $6.44 \mathrm{c}$ & $5.66 \mathrm{~d}$ & $0.67 c$ & $5.33 c$ & $0.55 d$ & $51.81 b$ & $51.10 \mathrm{~b}$ & $55.58 \mathrm{~b}$ & $52.90 \mathrm{~b}$ & $73.83 b$ & $53.85 b$ & $75.26 b$ \\
\hline $\mathrm{C}_{2}$ & $\begin{array}{c}6.66 \\
c\end{array}$ & $5.99 c$ & $7.66 b$ & $6.44 c$ & $0.67 c$ & $7.33 c$ & $0.89 c$ & $35.56 c$ & $41.11 \mathrm{c}$ & $40.50 c$ & $40.20 c$ & $56.12 \mathrm{c}$ & $38.26 c$ & $62.73 c$ \\
\hline $\mathrm{C}_{3}$ & $7.55 b$ & $7.55 b$ & $7.66 \mathrm{~b}$ & $7.22 \mathrm{~b}$ & $1.22 \mathrm{~b}$ & $8.00 \mathrm{~b}$ & $1.22 \mathrm{~b}$ & $23.32 \mathrm{~d}$ & $22.67 d$ & $31.96 \mathrm{~d}$ & $30.48 d$ & $32.54 d$ & $24.42 \mathrm{~d}$ & $39.25 d$ \\
\hline $\mathrm{C}_{4}$ & $\begin{array}{c}2.07 \\
e\end{array}$ & $2.11 e$ & $4.55 \mathrm{~d}$ & $2.66 e$ & $0.11 \mathrm{~d}$ & $2.55 e$ & $0.00 e$ & $78.57 \mathrm{a}$ & $78.19 a$ & 66.21a & $77.32 \mathrm{a}$ & $94.48 \mathrm{a}$ & 76.01a & $100.0 \mathrm{a}$ \\
\hline $\mathrm{S} \bar{x}$ & 0.13 & 0.13 & 0.07 & 0.11 & 0.02 & 0.17 & 0.03 & 0.35 & 0.46 & 0.43 & 0.49 & 1.68 & 1.14 & 0.93 \\
\hline CV (\%) & 6.72 & 6.88 & 2.67 & 5.16 & 5.70 & 7.87 & 9.32 & 2.81 & 3.57 & 3.29 & 3.66 & 9.80 & 8.94 & 5.01 \\
\hline
\end{tabular}

In a column, figures with the same letter do not differ significantly as per DMRT.

$C_{0}=$ No use of extract, $C_{1}=$ Aqueous extract of sorghum crop residues @ 1:20 ratio $(\mathrm{w} / \mathrm{v})$, $\mathrm{C}_{2}=$ Aqueous extract of sorghum crop residues @ 1:30 ratio $(\mathrm{w} / \mathrm{v}), \mathrm{C}_{3}=$ Aqueous extract of sorghum crop residues @ 1:40 ratio (w/v), $\mathrm{C}_{4}=$ Hand weeding

Combined effect of variety and aqueous extract of sorghum on number and percent inhibition on different weeds

For all the weed species $\mathrm{V}_{2} \mathrm{C}_{4}$ combination was found the lowest number of weed population. On the other hand highest percent inhibition was also found in the same combination. (Table 4)

Table 4. Combined effect of variety and aqueous extract of sorghum on number and percent inhibition on different weeds 


\begin{tabular}{|c|c|c|c|c|c|c|c|c|c|c|c|c|c|c|}
\hline \multicolumn{8}{|c|}{ Number of weed per quadrate $(25 \times 25) \mathrm{cm}^{2}$} & \multicolumn{7}{|c|}{$\%$ Inhibition } \\
\hline Weed name & \multirow[t]{2}{*}{ Bathua } & \multirow[t]{2}{*}{ Mutha } & \multirow[t]{2}{*}{ Biskatali } & \multirow[t]{2}{*}{ Durba } & \multirow{2}{*}{$\begin{array}{c}\text { Tit } \\
\text { begun }\end{array}$} & \multirow{2}{*}{ Shama } & \multirow[t]{2}{*}{ Angta } & \multirow[t]{2}{*}{ Bathua } & \multirow[t]{2}{*}{ Mutha } & \multirow[t]{2}{*}{ Biskatali } & \multirow[t]{2}{*}{ Durba } & \multirow{2}{*}{$\begin{array}{c}\text { Tit } \\
\text { begun }\end{array}$} & \multirow[t]{2}{*}{ Shama } & \multirow[t]{2}{*}{ Angta } \\
\hline Treatments & & & & & & & & & & & & & & \\
\hline $\mathrm{V}_{1} \mathrm{C}_{0}$ & $8.66 \mathrm{ab}$ & $8.00 \mathrm{abc}$ & $11.00 \mathrm{~b}$ & $8.33 b$ & $1.33 \mathrm{~b}$ & 10.33 & $2.00 \mathrm{a}$ & 0.00 & $0.00 \mathrm{~g}$ & $0.00 f$ & $0.00 \mathrm{~h}$ & 0.00 & 0.00 & $0.00 \mathrm{~g}$ \\
\hline $\mathrm{V}_{1} \mathrm{C}_{1}$ & $4.66 \mathrm{~g}$ & $4.667 f$ & $6.66 \mathrm{~g}$ & $6.33 e$ & $0.67 \mathrm{~d}$ & 5.33 & $0.67 d$ & 50.13 & $51.49 \mathrm{~b}$ & $54.61 \mathrm{c}$ & $51.40 \mathrm{~d}$ & 71.85 & 53.09 & $72.43 c$ \\
\hline $\mathrm{V}_{1} \mathrm{C}_{2}$ & 7.33d & $6.66 \mathrm{~d}$ & $6.67 \mathrm{~g}$ & $6.33 e$ & $0.67 \mathrm{~d}$ & 7.33 & $1.00 \mathrm{c}$ & 34.16 & $45.79 c$ & $40.23 d$ & $40.45 \mathrm{ef}$ & 50.32 & 37.77 & $60.32 d$ \\
\hline $\mathrm{V}_{1} \mathrm{C}_{3}$ & $7.66 \mathrm{~cd}$ & $7.66 \mathrm{bc}$ & $8.00 \mathrm{e}$ & $7.00 \mathrm{~cd}$ & $1.00 \mathrm{c}$ & 8.33 & $1.33 \mathrm{~b}$ & 23.08 & $25.68 e$ & $32.10 e$ & $30.13 \mathrm{~g}$ & 31.93 & 22.65 & $36.78 \mathrm{f}$ \\
\hline $\mathrm{V}_{1} \mathrm{C}_{4}$ & $2.23 \mathrm{~h}$ & $2.33 \mathrm{~g}$ & $4.33 \mathrm{j}$ & $2.66 \mathrm{gh}$ & $0.33 e$ & 2.66 & $0.00 \mathrm{f}$ & 76.54 & 77.31a & $65.32 b$ & $74.91 \mathrm{~b}$ & 83.44 & 74.66 & $100.0 \mathrm{a}$ \\
\hline $\mathrm{V}_{2} \mathrm{C}_{0}$ & $8.33 b c$ & 8.33ab & $11.67 \mathrm{a}$ & $9.00 \mathrm{a}$ & 2.33a & 9.00 & $2.00 \mathrm{a}$ & 0.00 & $0.00 \mathrm{~g}$ & $0.00 \mathrm{f}$ & $0.00 \mathrm{~h}$ & 0.00 & 0.00 & $0.00 \mathrm{~g}$ \\
\hline $\mathrm{V}_{2} \mathrm{C}_{1}$ & $5.00 \mathrm{fg}$ & $4.67 f$ & $6.67 \mathrm{~g}$ & $5.33 f$ & $0.67 \mathrm{~d}$ & 5.33 & $0.33 e$ & 53.29 & $51.76 \mathrm{~b}$ & $56.58 c$ & $55.14 \mathrm{c}$ & 75.04 & 55.18 & $80.52 b$ \\
\hline $\mathrm{V}_{2} \mathrm{C}_{2}$ & $6.33 e$ & $5.33 \mathrm{f}$ & 7.67ef & $6.33 e$ & $0.67 \mathrm{~d}$ & 7.00 & $0.67 d$ & 37.18 & $39.81 \mathrm{~d}$ & $40.62 \mathrm{~d}$ & $38.39 \mathrm{f}$ & 60.30 & 39.05 & $64.17 \mathrm{~d}$ \\
\hline $\mathrm{V}_{2} \mathrm{C}_{3}$ & $7.66 \mathrm{~cd}$ & $7.33 \mathrm{c}$ & 7.67ef & $7.33 c$ & $1.33 \mathrm{~b}$ & 7.66 & $1.00 \mathrm{c}$ & 23.68 & $21.20 f$ & $31.44 e$ & $29.47 \mathrm{~g}$ & 35.41 & 25.75 & $46.26 e$ \\
\hline $\mathrm{V}_{2} \mathrm{C}_{4}$ & $2.00 \mathrm{~h}$ & $2.00 \mathrm{~g}$ & $4.00 \mathrm{j}$ & $2.33 \mathrm{~h}$ & $0.00 \mathrm{f}$ & 2.33 & $0.00 \mathrm{f}$ & 80.72 & $79.20 \mathrm{a}$ & $69.44 a$ & $79.78 \mathrm{a}$ & 100.0 & 77.84 & $100.0 \mathrm{a}$ \\
\hline $\mathrm{V}_{3} \mathrm{C}_{0}$ & $9.33 \mathrm{a}$ & $8.66 a$ & $10.33 \mathrm{c}$ & $9.00 \mathrm{a}$ & 2.33a & 9.33 & $2.00 \mathrm{a}$ & 0.00 & $0.00 \mathrm{~g}$ & $0.00 \mathrm{f}$ & $0.00 \mathrm{~h}$ & 0.00 & 0.00 & $0.00 \mathrm{~g}$ \\
\hline $\mathrm{V}_{3} \mathrm{C}_{1}$ & 5.66ef & $4.66 f$ & $6.00 \mathrm{~h}$ & $5.33 f$ & $0.67 d$ & 5.33 & $0.67 d$ & 52.01 & $50.19 b$ & $55.56 \mathrm{c}$ & $52.07 d$ & 74.61 & 53.28 & $72.83 c$ \\
\hline $\mathrm{V}_{3} \mathrm{C}_{2}$ & $6.33 e$ & $6.00 e$ & $8.66 \mathrm{~d}$ & 6.66de & $0.67 \mathrm{~d}$ & 7.66 & $1.00 \mathrm{c}$ & 35.33 & $37.74 \mathrm{~d}$ & $40.66 d$ & $41.80 e$ & 57.75 & 37.97 & $63.71 \mathrm{~d}$ \\
\hline $\mathrm{V}_{3} \mathrm{C}_{3}$ & $7.33 \mathrm{~d}$ & $7.66 b c$ & $7.33 \mathrm{f}$ & $7.33 c$ & $1.33 \mathrm{~b}$ & 8.00 & $1.33 b$ & 23.21 & $21.09 f$ & $32.35 e$ & $31.83 \mathrm{~g}$ & 30.29 & 24.85 & $34.72 f$ \\
\hline $\mathrm{V}_{3} \mathrm{C}_{4}$ & $2.00 \mathrm{~h}$ & $2.00 \mathrm{~g}$ & $5.33 \mathrm{i}$ & $3.00 \mathrm{~g}$ & $0.00 \mathrm{f}$ & 2.66 & $0.00 \mathrm{f}$ & 78.45 & $78.06 a$ & $63.88 \mathrm{~b}$ & $77.28 \mathrm{~b}$ & 100.0 & 75.53 & $100.0 \mathrm{a}$ \\
\hline$S \bar{x}$ & 0.25 & 0.29 & 0.12 & 0.18 & 0.03 & 0.29 & 0.05 & 0.61 & 0.79 & 0.74 & 0.85 & 2.90 & 1.99 & 1.60 \\
\hline$\overline{\mathrm{CV}}(\%)$ & 6.72 & 6.88 & 2.67 & 5.16 & 5.70 & 7.87 & 9.32 & 2.81 & 3.57 & 3.29 & 3.66 & 9.80 & 8.94 & 5.01 \\
\hline
\end{tabular}

In a column, figures with the same letter do not differ significantly as per DMRT.

$\mathrm{V}_{1}=$ BARI Gom-19 (Sourav), $\mathrm{V}_{2}=$ BARI Gom-21 (Shatabdi), $\mathrm{V}_{3}=$ BARI Gom-24 (Prodip)

$\mathrm{C}_{0}=$ No use of extract, $\mathrm{C}_{1}=$ Aqueous extract of sorghum crop residues @ 1:20 ratio (w/v), $\mathrm{C}_{2}=$ Aqueous extract of sorghum crop residues @ 1:30 ratio (w/v), $\mathrm{C}_{3}=$ Aqueous extract of sorghum crop residues @ 1:40 ratio (w/v), $\mathrm{C}_{4}=$ Hand weeding

\section{Effect of variety on yield and yield contributing characters of wheat}

Varietal effect on yield and yield contributing characters of wheat showed the significant effect. Highest plant height, higher number of total tillers and effective tillers hill ${ }^{-1}$, higher number of grain spike $e^{-1}$, higher number of filled grain spike $e^{-1}$, highest straw yield and highest harvest index was found in $V_{2}$ (BARI Gom-21) variety (Table 5). The highest grain yield $\left(3.84 \mathrm{t} \mathrm{ha}^{-1}\right)$ was obtained in BARI Gom-21 followed by BARI Gom-24 (3.69 t ha $\left.{ }^{-1}\right)$. (Fig. 1)

Table 5. Effect of variety on yield and yield contributing characters of wheat

\begin{tabular}{lccccccccc}
\hline Varieties & $\begin{array}{c}\text { Plant } \\
\text { height } \\
(\mathrm{cm})\end{array}$ & $\begin{array}{c}\text { No. of } \\
\text { total } \\
\text { tillers } \\
\text { hill }^{-1}\end{array}$ & $\begin{array}{c}\text { No. of } \\
\text { effective } \\
\text { tillers } \\
\text { hill }^{-1}\end{array}$ & $\begin{array}{c}\text { Panicle } \\
\text { length } \\
(\mathrm{cm})\end{array}$ & $\begin{array}{c}\text { No. of } \\
\text { grains } \\
\text { spike }\end{array}$ & $\begin{array}{c}\text { Filled } \\
\text { grain } \\
\text { spike }\end{array}$ & $\begin{array}{c}1000- \\
\text { grain } \\
\text { weight } \\
(\mathrm{gm})\end{array}$ & $\begin{array}{c}\text { Straw } \\
\text { yield } \\
\left(\mathrm{t} \mathrm{ha} \mathrm{h}^{-1}\right)\end{array}$ & $\begin{array}{c}\text { Harvest } \\
\text { index } \\
(\%)\end{array}$ \\
\hline $\mathrm{V}_{1}$ & $88.71 \mathrm{c}$ & $4.02 \mathrm{~b}$ & $3.16 \mathrm{~b}$ & 10.29 & 42.23 & $15.22 \mathrm{~b}$ & $55.18 \mathrm{a}$ & $4.01 \mathrm{~b}$ & $45.14 \mathrm{~b}$ \\
$\mathrm{~V}_{2}$ & $95.51 \mathrm{a}$ & $4.13 \mathrm{a}$ & $3.35 \mathrm{a}$ & 10.46 & 43.36 & $15.75 \mathrm{a}$ & $53.60 \mathrm{ab}$ & $4.36 \mathrm{a}$ & $46.75 \mathrm{a}$ \\
$\mathrm{V}_{3}$ & $91.24 \mathrm{~b}$ & $4.07 \mathrm{ab}$ & $3.25 \mathrm{~b}$ & 10.36 & 42.76 & $15.42 \mathrm{ab}$ & $52.39 \mathrm{~b}$ & $4.35 \mathrm{a}$ & $45.57 \mathrm{~b}$ \\
\hline $\mathrm{S} \overline{\mathrm{x}}$ & 0.761 & 0.023 & 0.032 & 0.105 & 0.385 & 0.141 & 0.606 & 0.037 & 0.348 \\
\hline $\mathrm{CV}(\%)$ & 3.21 & 2.14 & 3.85 & 3.92 & 3.48 & 3.53 & 4.37 & 3.40 & 2.94 \\
\hline
\end{tabular}

In a column, figures with the same letter do not differ significantly as per DMRT.

$\mathrm{V}_{1}=$ BARI Gom-19 (Sourav), $\quad \mathrm{V}_{2}=$ BARI Gom-21 (Shatabdi), $\mathrm{V}_{3}=$ BARI Gom-24 (Prodip) 


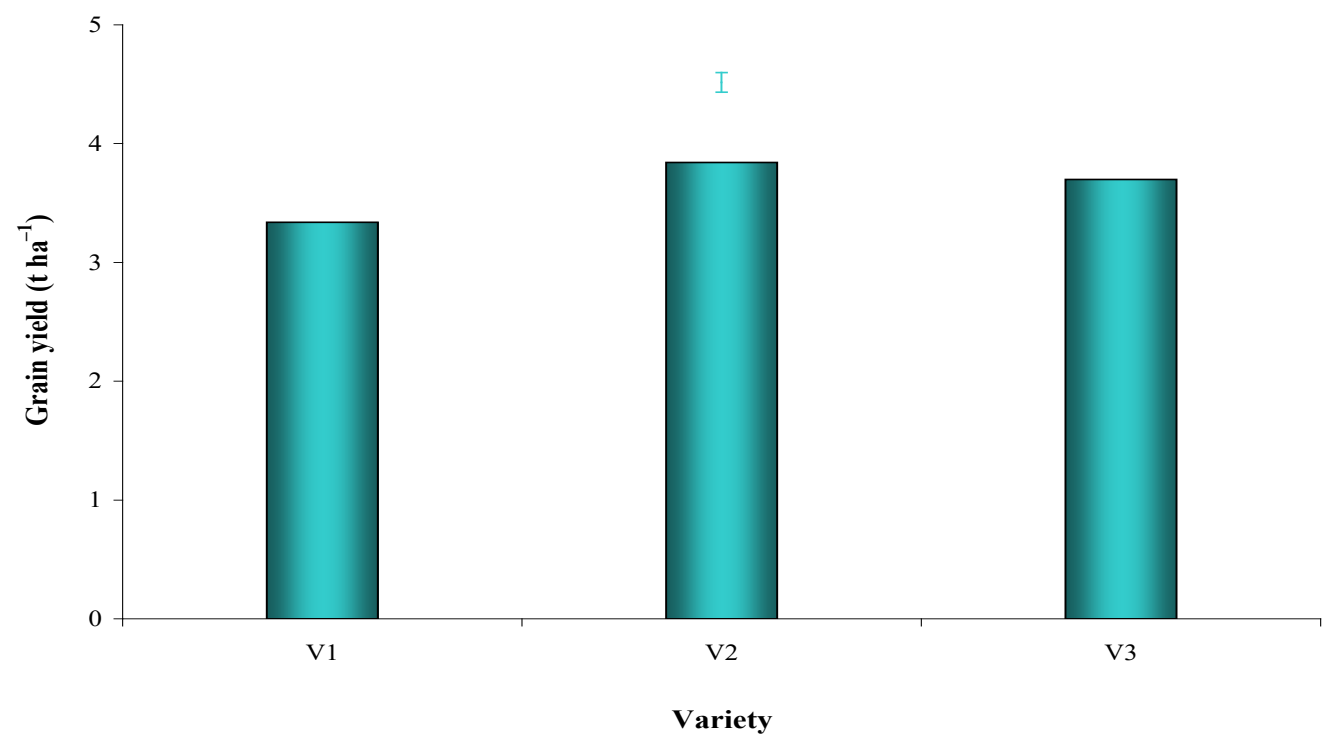

Fig. 1. Grain yield as influenced by variety (Bar represents standard error mean) Here, $V_{1}=$ BARI Gom-19 (Sourav), $V_{2}=$ BARI Gom-21 (Shatabdi), $V_{3}=$ BARI Gom-24

(Prodip)

Effect of aqueous extract of sorghum crop residues on yield and yield contributing characters of wheat

Aqueous extract of sorghum crop residues had also significant effect on yield and yield contributing characters. The highest grain yield $\left(3.97 \mathrm{t} \mathrm{h} \mathrm{a}^{-1}\right)$ was produced by $\mathrm{C}_{4}$ treatment, followed by $\mathrm{C}_{1}\left(3.88 \mathrm{t} \mathrm{ha}^{-1}\right)$ and lowest one $\left(2.61 \mathrm{t} \mathrm{ha}^{-1}\right)$ was produced by $\mathrm{C}_{0}$ (no use of extract) treatment due to the production of higher number of effective tillers hill ${ }^{-1}$, higher number of grain spike ${ }^{-1}$, higher number of filled grain spike $e^{-1}$. The lowest grain yield was produced in $\mathrm{C}_{0}$ treatment. (Table 6 and Fig. 2). Uddin and Pyon (2010) also reported the similar results, where crop residues influenced in crop performance.

Table 6. Effect of aqueous extract of sorghum crop residues on yield and yield contributing characters of wheat

\begin{tabular}{|c|c|c|c|c|c|c|c|c|c|}
\hline Residues & $\begin{array}{c}\text { Plant } \\
\text { height } \\
(\mathrm{cm})\end{array}$ & $\begin{array}{l}\text { No. } \\
\text { of } \\
\text { total } \\
\text { tillers } \\
\text { hill }^{-1} \\
\end{array}$ & $\begin{array}{l}\text { No. of } \\
\text { effective } \\
\text { tillers } \\
\text { hill }^{-1}\end{array}$ & $\begin{array}{l}\text { Panicle } \\
\text { length } \\
\text { (cm) }\end{array}$ & $\begin{array}{l}\text { No. of } \\
\text { grains } \\
\text { spike }\end{array}$ & $\begin{array}{c}\text { Filled } \\
\text { grain } \\
\text { spike }\end{array}$ & $\begin{array}{c}1000 \\
\text { grain } \\
\text { weight } \\
\text { (gm) }\end{array}$ & $\begin{array}{l}\text { Straw } \\
\text { yield } \\
\left(\mathrm{t} \quad \mathrm{ha}^{-1}\right)\end{array}$ & $\begin{array}{c}\text { Harvest } \\
\text { index }(\%)\end{array}$ \\
\hline $\mathrm{C}_{0}$ & $82.27 \mathrm{~d}$ & $3.46 \mathrm{~d}$ & $2.70 e$ & $9.57 c$ & $39.72 d$ & $13.28 \mathrm{~d}$ & $51.27 b$ & $3.51 c$ & $42.12 b$ \\
\hline $\mathrm{C}_{1}$ & $94.92 \mathrm{~b}$ & $4.21 \mathrm{~b}$ & $3.41 b$ & $10.58 \mathrm{ab}$ & $44.08 \mathrm{~b}$ & $16.24 \mathrm{~b}$ & $53.27 \mathrm{~b}$ & $4.29 \mathrm{~b}$ & $47.48 \mathrm{a}$ \\
\hline $\mathrm{C}_{2}$ & $92.75 b c$ & $4.13 b$ & $3.29 c$ & $10.49 b$ & $42.62 c$ & $15.42 \mathrm{c}$ & $53.44 b$ & $4.40 \mathrm{ab}$ & $46.60 \mathrm{a}$ \\
\hline $\mathrm{C}_{3}$ & $91.02 \mathrm{c}$ & $4.02 \mathrm{c}$ & $3.13 \mathrm{~d}$ & $10.28 b$ & $41.02 \mathrm{~d}$ & $15.05 c$ & $52.96 b$ & 4.43ab & $46.23 a$ \\
\hline $\mathrm{C}_{4}$ & $98.14 a$ & $4.54 \mathrm{a}$ & $3.72 \mathrm{a}$ & $10.92 \mathrm{a}$ & $46.54 a$ & $17.34 a$ & $57.66 a$ & $4.54 \mathrm{a}$ & $46.67 a$ \\
\hline $\mathrm{S} \overline{\mathrm{x}}$ & 0.983 & 0.030 & 0.042 & 0.135 & 0.497 & 0.182 & 0.782 & 0.048 & 0.450 \\
\hline $\begin{array}{l}\text { Level of } \\
\text { significance }\end{array}$ & $* *$ & $* *$ & $* *$ & $* *$ & $* *$ & $\% *$ & $* *$ & $* *$ & $* *$ \\
\hline CV (\%) & 3.21 & 2.14 & 3.85 & 3.92 & 3.48 & 3.53 & 4.37 & 3.40 & 2.94 \\
\hline
\end{tabular}

** =Significant at $1 \%$ level of probability.

$\mathrm{C}_{0}=$ No use of extract, $\mathrm{C}_{1}=$ Aqueous extract of sorghum crop residues @ 1:20 ratio (w/v), $\mathrm{C}_{2}=$ Aqueous extract of sorghum crop residues @ $1: 30$ ratio (w/v), $\mathrm{C}_{3}=$ Aqueous extract of sorghum crop residues @ 1:40 ratio (w/v), $\mathrm{C}_{4}=$ Hand weeding. 


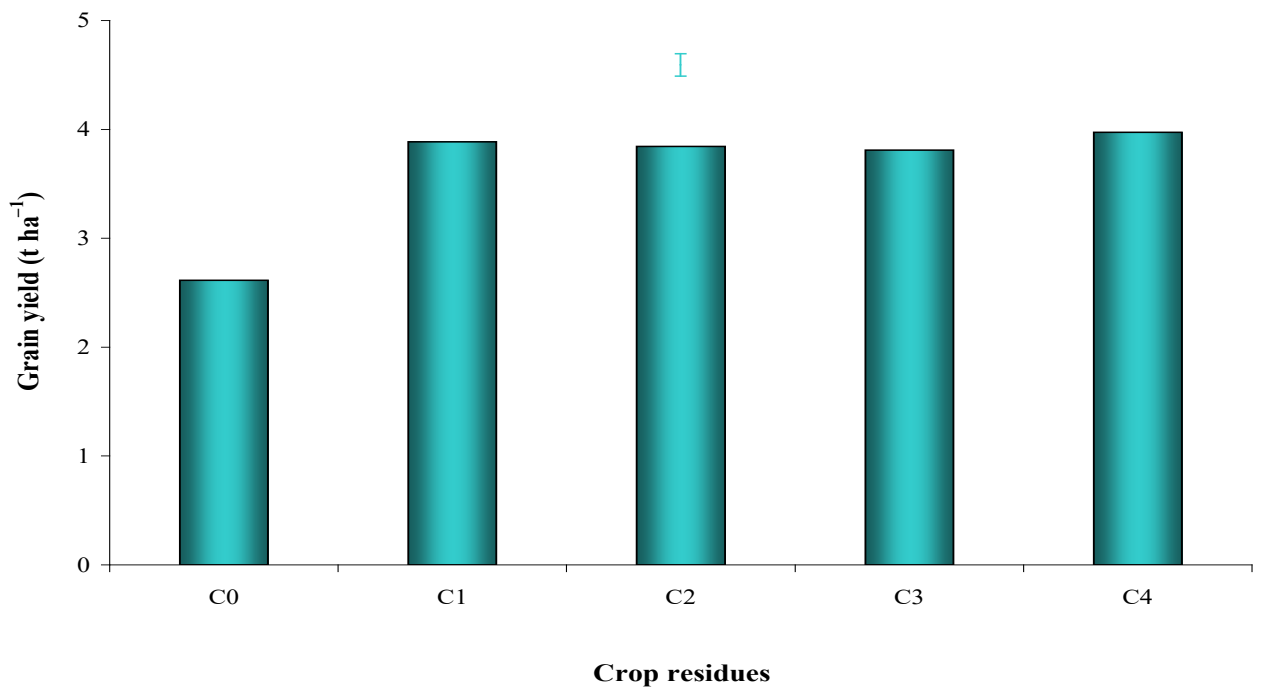

Fig. 2. Grain yield as influenced by aqueous extract of sorghum crop residues (Bar represents standard error mean)

$C_{0}=$ No use of extract, $C_{1}=$ Aqueous extract of sorghum crop residues @ $1: 20$ ratio $(\mathrm{w} / \mathrm{v})$, $\mathrm{C}_{2}=$ Aqueous extract of sorghum crop residues @ 1:30 ratio (w/v), $\mathrm{C}_{3}=$ Aqueous extract of sorghum crop residues @ 1:40 ratio (w/v), $\mathrm{C}_{4}=$ Hand weeding

Combined effects of variety and aqueous extract of sorghum crop residues on yield and yield contributing characters of wheat

Yield and yield contributing characters like straw yield and grain yield were significantly affected by the interaction between variety and crop residues. $\mathrm{V}_{2} \mathrm{C}_{4}$ combination showed the maximum result which was followed by $\mathrm{V}_{3} \mathrm{C}_{1}$. (Table 7)

Table 7. Combined effects of variety and aqueous extract of sorghum crop residues on yield and yield contributing characters of wheat

\begin{tabular}{|c|c|c|c|c|c|c|c|c|c|c|}
\hline $\begin{array}{l}\text { Variety x } \\
\text { Residues }\end{array}$ & $\begin{array}{l}\text { Plant } \\
\text { height } \\
\text { (cm) }\end{array}$ & $\begin{array}{c}\text { No. of } \\
\text { total } \\
\text { tillers } \\
\text { hill }^{-1} \\
\end{array}$ & $\begin{array}{l}\text { No. of } \\
\text { effective } \\
\text { tillers } \\
\text { hill }^{-1}\end{array}$ & $\begin{array}{c}\text { Spike } \\
\text { length } \\
\text { (cm) }\end{array}$ & $\begin{array}{c}\text { No. } \\
\text { of } \\
\text { grain } \\
\text { spike }\end{array}$ & $\begin{array}{l}\text { No of } \\
\text { spikelet } \\
\text { spike }^{-1}\end{array}$ & $\begin{array}{l}1000 \\
\text { grain } \\
\text { weight } \\
(\mathrm{gm})\end{array}$ & $\begin{array}{l}\text { Grain } \\
\text { yield } \\
\left(\mathrm{t}^{\mathrm{ha}} \mathrm{ha}^{-1}\right)\end{array}$ & $\begin{array}{c}\text { Straw } \\
\text { yield } \\
\left(\mathrm{t} \mathrm{ha}^{-1}\right)\end{array}$ & $\begin{array}{l}\text { Harvest } \\
\text { index } \\
(\%)\end{array}$ \\
\hline $\mathrm{V}_{1} \mathrm{C}_{0}$ & 80.73 & 3.40 & 2.57 & 9.55 & 39.26 & 12.94 & 55.07 & $2.16 \mathrm{i}$ & $3.11 \mathrm{~g}$ & 41.00 \\
\hline & & & & 10.48 & 43.64 & & & 3.63ef & & \\
\hline $\mathrm{V}_{1} \mathrm{C}_{2}$ & 89 & & 3. & 0.41 & 42.11 & & & & & \\
\hline $\mathrm{V}_{1} \mathrm{C}_{3}$ & $88 .^{\circ}$ & & & & & & & & & \\
\hline $\mathrm{V}_{1} \mathrm{C}_{4}$ & 93.5 & & & 10.81 & & & & $3.77 \mathrm{de}$ & & \\
\hline $\mathrm{V}_{2} \mathrm{C}_{0}$ & 83.7 & & & 9.59 & 40.04 & & & & & 43. \\
\hline & 99.69 & 4.2 & & 10.72 & 44.86 & 16. & 53 & 4.0 & & \\
\hline $\mathrm{V}_{2} \mathrm{C}_{2}$ & & & & & 42.95 & & 52.87 & & & \\
\hline $\mathrm{V}_{2} \mathrm{C}_{3}$ & 9 & & & 0.35 & 41.45 & 15. & 52.18 & 4.0 & & 46. \\
\hline $\mathrm{V}_{2} \mathrm{C}_{4}$ & 10 & & & 11.04 & 47.47 & & & & & \\
\hline & & & & 9.5 & & & & & & 41.22 \\
\hline $\mathrm{V}_{3} \mathrm{C}_{1}$ & & & 3.37 & 10.56 & 43.74 & & & 3.9 & & \\
\hline & & & & & & & & & & \\
\hline & 90.33 & & 3.20 & 10.26 & 41.14 & & & & & \\
\hline & & & & & & & 57.09 & 4.02abc & 4.57ab & 46.82 \\
\hline $\mathrm{S} \overline{\mathrm{x}}$ & 1.70 & 0.050 & 0.072 & 0.234 & 0.861 & 0.315 & 1.35 & 0.055 & 0.084 & 0.779 \\
\hline $\begin{array}{l}\text { Level of } \\
\text { significance }\end{array}$ & NS & NS & NS & NS & NS & NS & NS & * & ** & NS \\
\hline CV (\%) & 3.21 & 2.14 & 3.85 & 3.92 & 3.48 & 3.53 & 4.37 & 2.61 & 3.40 & 2.94 \\
\hline
\end{tabular}

** $=$ Significant at $1 \%$ level of probability, ${ }^{*}=$ Significant at $5 \%$ level of probability, NS $=$ Non significant 
$\mathrm{V}_{1}=$ BARI Gom-19 (Sourav), $\mathrm{V}_{2}=$ BARI Gom-21 (Shatabdi), $\mathrm{V}_{3}=$ BARI Gom-24 (Prodip)

$C_{0}=$ No use of extract, $C_{1}=$ Aqueous extract of sorghum crop residues @ 1:20 ratio (w/v),

$\mathrm{C}_{2}=$ Aqueous extract of sorghum crop residues @ 1:30 ratio (w/v), $\mathrm{C}_{3}=$ Aqueous extract of sorghum crop residues @ 1:40 ratio (w/v), $C_{4}=$ Hand weeding

\section{Conclusion}

From the above results it was found that the wheat var. BARI Gom-21 with $\mathrm{C}_{4}$ (hand weeding) treatment exhibited the superior effect and BARI Gom-21 with $\mathrm{C}_{1}$ showed quite close result with it. Results of the present study reveal that application of aqueous extract of sorghum crop residues has positive effect on yield for most of the studied traits. It also shows that aqueous extract of sorghum crop residues have herbicidal activity for suppressing weed growth and there is immense prospect of sorghum crop residues as a weed management tool. Therefore, aqueous extract of sorghum crop residues could be a prospective source of weed control tool for crop production in modern agricultural science which minimizes cost of production, environmental pollution, unsafe agricultural production, human health concerns, soil sickness and depletion of crop diversity.

\section{References}

Ahmed, G.J.U., M.K.A. Bhuiyan, C.R. Riches, M. Mortimer and D. Jhonson 2005. Farmer's participatory studies of integrated weed management system for intensified lowland. Proceeding of the 8th Biennial Agronomy Convention. Bangladesh Agron. J. 31-32.

BBS (Bangladesh Bureau of Statistics) 2015. Statistical Year Book of Bangladesh. Bangladesh Bureau, Statistics Division, Ministry of Planning, Government of the People's Republic of Bangladesh, Dhaka, Bangladesh. pp. 78-79.

Belz, R.G. 2004. Evaluation of allelopathic traits in Triticum spp L. and Secale cereal L.PhD Thesis, University of Hohenheim, Stuttgart, Germany.

Khan, T.D., M.I. Chung, T.D. Xuan and S. Tawata 2005. The exploitation of crop allelopathy in sustainable agricultural production. J. Agron. Crop Sci. 191: 172-184.

Prasad, K. and A. Rafey 1995. Effect of integrated weed management on weed growth, nutrient uptake, economics and energetics in rainfed upland rice (Oryza sativa). Indian J. Agr. Sci. 65: 260-264.

Sathyamoorthy, N.K., S. Mahendran, R. Babu and T. Ragavan 2004. Effect of integrated weed management practices on total weed dry weight, nutrient removal of weeds in rice-rice wet seedbed system. J. Agron. 3: 263-267.

Uddin, M.R. and J.Y. Pyon 2010. Herbicidal Activity of Rotation Crop Residues on Weeds and Selectivity to Crops. J. Agril. Sci. 37: 1-6. 